\title{
Monte Carlo Simulations of Natural Uranium Setups Irradiated With Relativistic Deuterons by Means of MCNPX Code
}

\author{
M. Suchopár ${ }^{1 a b}$, V. Wagner ${ }^{a}$, J. Vrzalováab ${ }^{\text {, O. Svoboda }}$ \\ ${ }^{a}$ Nuclear Physics Institute of ASCR \\ $25068 \check{R}$ ž near Prague, Czech Republic \\ ${ }^{b}$ Faculty of Nuclear Sciences and Physical Engineering, Czech Technical University in Prague \\ Břehová 7, 11519 Prague, Czech Republic \\ E-mail: suchopar@ujf.cas.cz, wagner@ujf.cas.cz, vrzalova@ujf.cas.cz, \\ svobodalujf.cas.cz
}

\section{J. Adam, A. Baldin, W. Furman, M. Kadykov, A. Solnyshkin, V. Tsoupko-Sitnikov, S. Tyutyunnikov, N. Vladimirovna, L. Závorka ${ }^{\text {b }}$}

Joint Institute for Nuclear Research

Joliot Curie str. 6, 1419980 Dubna, Moscow region, Russia

E-mail: iadamejinr.ru, Anton.Baldinesunhe.jinr.ru, furmanejinr.ru,

kadykovajinr.ru, solnesunhe.jinr.ru, vtsoupkoljinr.ru,

tsiesunhe.jinr.ru, vladimirovnaljinr.ru, zavorkaljinr.ru

\section{Chilap, A. Chinenov}

Center for Physical and Technical Projects "Atomenergomash"

Moscow, Russia

E-mail: chilap@cftp-aem.ru, chinenov@cftp-aem.ru

The collaboration Energy and Transmutation of Radioactive Waste has used different set-ups consisting of lead, uranium and graphite irradiated by relativistic protons and deuterons to study transmutation of radioactive materials by produced neutrons. Such studies are intended for providing important data serving for the construction of larger-scale Accelerator-Driven Systems. The most recent set-up named QUINTA, consisting of natural uranium target and lead blanket, was irradiated by deuteron beams in the energy range between 1 and $8 \mathrm{GeV}$ in the last three accelerator runs at JINR Nuclotron in 2011 and 2012. Monte Carlo simulations of the target assembly QUINTA concerning overall neutron production as well as spatial and energy distribution of the neutron field inside the set-up have been made using the MCNPX code and they have been compared with experimental data obtained by activation method.

XXI International Baldin Seminar on High Energy Physics Problems September 10-15, 2012

JINR, Dubna, Russia

Speaker 


\section{Introduction}

Experiments with the Quinta set-up have been performed within the framework of the Energy plus Transmutation of Radioactive Waste international collaboration. This workgroup is focused on research of Accelerator-Driven Systems based on a subcritical nuclear reactor driven by an external spallation neutron source. Irradiations of the Quinta set-up were carried out at the JINR Nuclotron accelerator facility by 1,4 , and $8 \mathrm{GeV}$ deuteron beam.

The main objective of this paper is to assess the accuracy of the respective Monte Carlo simulations, which use various models of spallation reactions and cross-section libraries of neutron and proton induced reactions. The MCNPX code has been used for the simulation of the interaction of primary beam in the target, the course of spallation reactions and the propagation of high-energy neutrons through the studied target-blanket installation. This paper is aimed at calculation of the overall neutron production in the whole assembly using different high-energy physics models. It further describes measurement of spatial distribution of produced neutron field in the experimental system by activation method and comparison with results originating from simulation.

\section{Description of the Quinta set-up}

The Quinta set-up consists of a natural uranium target-blanket and an additional lead blanket-shielding. The target-blanket part of the Quinta set-up is composed of five sections having $114 \mathrm{~mm}$ in length and separated by $17 \mathrm{~mm}$ gaps. The length of the inner set-up is $655 \mathrm{~mm}$, while the length of the whole assembly with the lead shielding is $900 \mathrm{~mm}$. The mass of the target-blanket is around $540 \mathrm{~kg}$ and the total mass of uranium in the set-up approximately $512 \mathrm{~kg}$. Each section is encased in a container with a hexagonal cross-section. The sections are enclosed in aluminium plates from all sides. The target-blanket of the Quinta set-up is filled with natural uranium rods (diameter of $36 \mathrm{~mm}$, length of $104 \mathrm{~mm}$, and weight of $1.72 \mathrm{~kg}$ ) with aluminium cladding of thicknesses $1 \mathrm{~mm}$. The first section has a central hole for the beam entrance with a diameter of $80 \mathrm{~mm}$. The remaining four sections are identical and entirely filled with uranium rods distributed in a hexagonal lattice. The first section contains 54 uranium rods with a total mass of $92 \mathrm{~kg}$, while the rest of the sections contain 61 uranium rods with a total mass of $105 \mathrm{~kg}$ each. The assembly is mounted on an aluminium plate of dimensions $700 \times 400 \times 16 \mathrm{~mm}$. The target-blanket is surrounded by a lead blanket serving for additional neutron multiplication and back-scattering to the central part of the uranium assembly. The lead shielding has $100 \mathrm{~mm}$ in thickness. In the front part, there is an entrance window in the shielding with dimensions of 150x150 mm. In the gaps between the sections, in front of the first section and behind the last section, there are six aluminium detector plates of thickness $2 \mathrm{~mm}$, which are removable through mounting pits in the shielding. Marks on concentric circles of radius 40,80 and $120 \mathrm{~mm}$ can be found on the plates. In the upper half of the plates, there are holes for placement of up to six cadmium containers for activation samples (length of $10 \mathrm{~mm}$, 
diameter of $15 \mathrm{~mm}$ ), three on radius $40 \mathrm{~mm}$ and another three on radius $120 \mathrm{~mm}$. Additional activation detectors can be placed directly on the front or rear side of the plates.

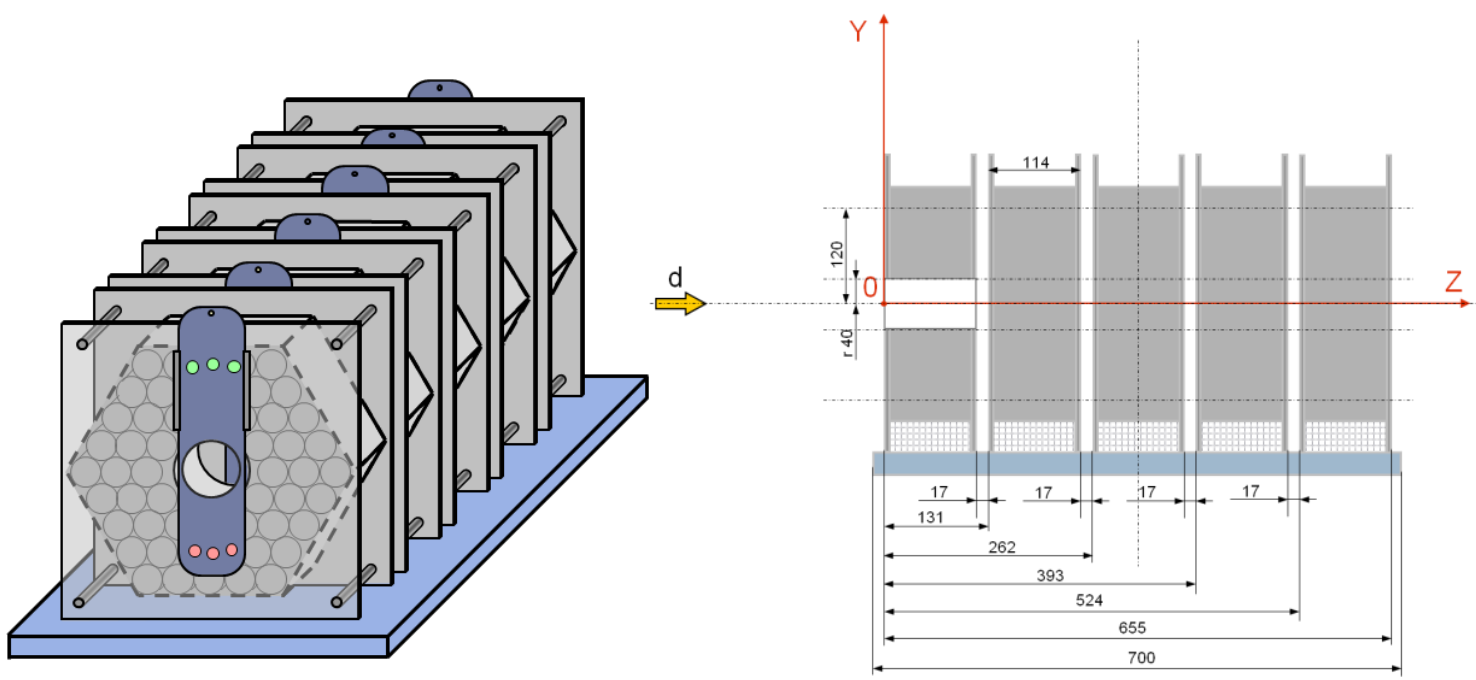

Fig. 1. General front (left) and cross-sectional side (right) view of the Quinta set-up

\section{Neutron activation method}

\subsection{Beam parameters determination}

The Quinta set-up was irradiated at the Nuclotron accelerator with 1 and $4 \mathrm{GeV}$ deuteron beam in December 2011 and with 1, 4 and $8 \mathrm{GeV}$ deuteron beam in March 2012. The total beam intensity was determined by the activation analysis method using activation foils made of aluminium. The integral deuteron beam flux was measured by aluminium foils placed on a holder between the beam tube window and the first of the ionization chambers located in front of the target. A square foil of size $100 \times 100 \mathrm{~mm}$ was used for this purpose. In the process of irradiation, stable isotope ${ }^{27} \mathrm{Al}$ was transmuted by $(\mathrm{d}, 3 \mathrm{p} 2 \mathrm{n})$ reaction into radioactive isotope ${ }^{24} \mathrm{Na}$. The yield (the number of nuclei activated in the foil during the whole period of the irradiation) of produced gamma-radioactive nuclei was determined using gamma spectroscopy.

The position and shape of the beam profile was determined independently by a set of copper activation foils and solid state nuclear track detectors placed on the front side of the setup. For the measurement of the beam position before impact on the target, a copper foil of size $80 \times 80 \mathrm{~mm}$ was used. The foil was cut after the irradiation into 16 pieces of size $20 \times 20 \mathrm{~mm}$ and every piece was measured separately.

\subsection{Spectra evaluation method}

To analyze the gamma-ray spectra and to determine net peak areas, the computer program DEIMOS32 was used. Corrections for decay, gamma line intensity, detector efficiency, dead time, possible coincidence effects, beam instability during irradiation, nonpoint-like emitters, 
and self-absorption correction were applied to obtain the total number of nuclei of certain isotope. The yield was then normalized to one gram of activation foil mass and to one primary beam deuteron.

\section{Monte Carlo simulation}

\subsection{Spallation process}

The Monte Carlo simulations of neutron multiplicity, spatial and energy distribution of the neutron field inside the Quinta set-up, and activation reactions in the foils were performed with the MCNPX code version 2.7. Standard cross-section libraries ENDF/B VII.0 (up to $200 \mathrm{MeV}$ ) included in the MCNPX code package were used for the simulations.

The spallation reaction is simulated in three phases: intra-nuclear cascade, pre-equilibrium emission and evaporation stage. The intra-nuclear cascade (INC) can be described by Bertini, Isabel, or INCL4 model, or CEM03 stand-alone model. The multistage pre-equilibrium exciton model (included in INC models) is used for the pre-equilibrium emission of particles. The equilibrium emission of particles can be described by Dresner or ABLA evaporation models.

\subsection{Neutron multiplicity}

A detailed geometric model of the Quinta set-up was developed for the purpose of MCNPX simulations. Nucleons and charged pions were taken into account in the computations. Multiplicity (the number of produced neutrons per one incident deuteron, calculated as a sum of the number of neutrons escaped from the set-up and the number of neutrons captured in the setup) of the Quinta set-up was determined using different combinations of INC and evaporation models.

All seven possible combinations of INC models and evaporation models (Bertini+Dresner, Isabel+Dresner, INCL+Dresner, Bertini+ABLA, Isabel+ABLA, INCL+ABLA, CEM) were tested in combination with FLUKA or LAQGSM for higher energies in order to assess if these combinations of built-in models predict similar neutron multiplicity.

It can be noticed in figure 2 that the additional lead blanket slightly increases the neutron flux evenly in the whole set-up and that the first and last section have only minor influence on the overall neutron production in the system and decrease the neutron flux in the longitudinal direction in the parts near the front and back end of the set-up.

The difference between various combinations of models is within $15 \%$ for the set-up without lead blanket and within $10 \%$ for the set-up with lead blanket. The additional lead blanket contributes approximately 8-10\% to the amount of neutrons created in the set-up. In general, the combinations with ABLA evaporation model give higher neutron multiplicity than the combinations with Dresner evaporation model. The highest neutron multiplicities generally result from CEM03 and INCL-ABLA models. 
The graph in figure 4 shows that the models calculate well up to the incident beam energy of $2 \mathrm{GeV}$ but for higher energies a minor progressive decrease of the simulated multiplicity per $\mathrm{GeV}$ can be observed when compared to the experimental multiplicity which should stay constant in this energy region.
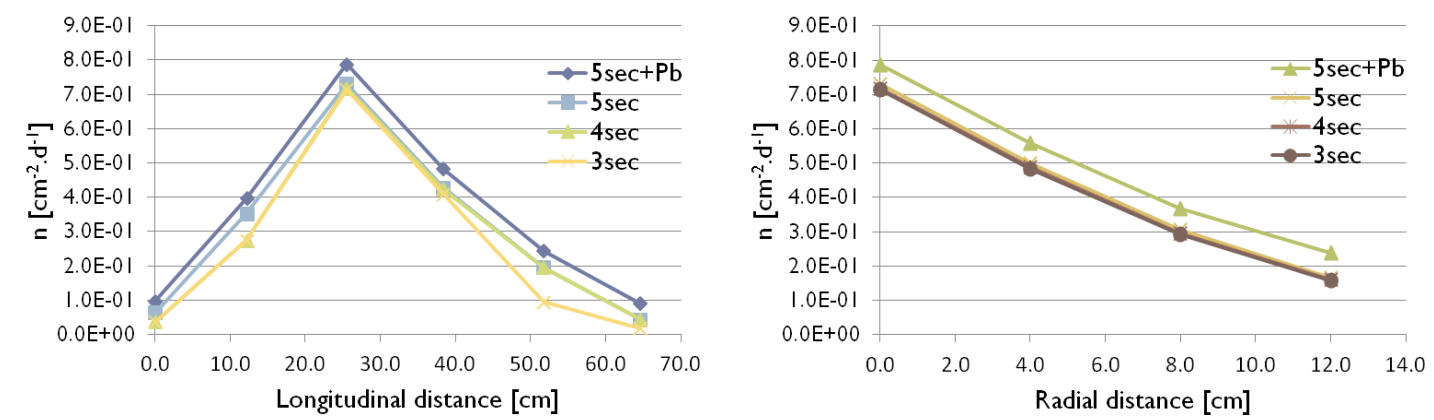

Fig. 2. Distribution of neutron flux in longitudinal (left) and radial (right) direction for the bare set-up and for the set-up with lead blanket
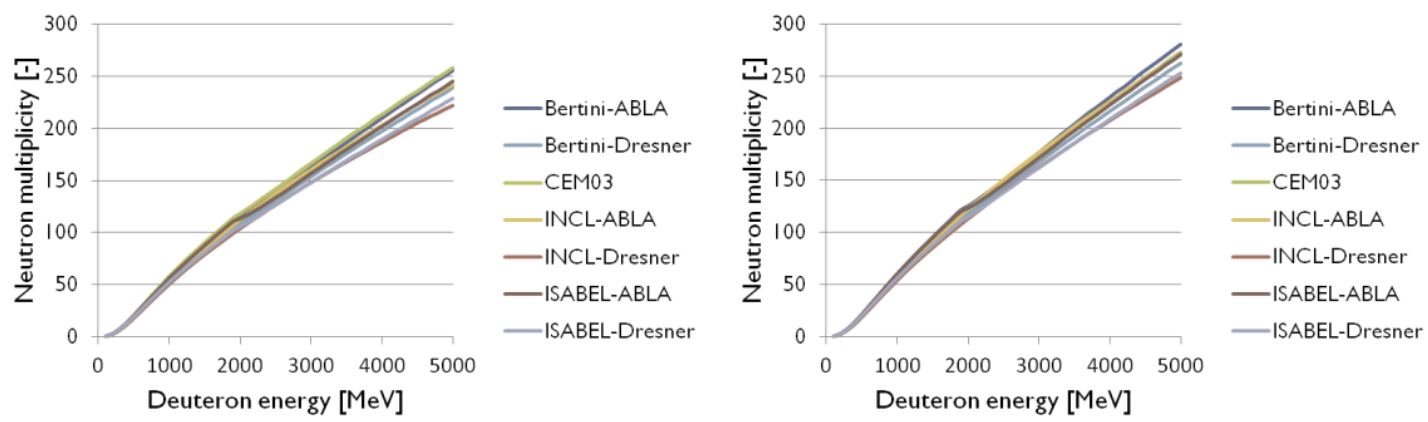

Fig. 3. Neutron multiplicity for the bare set-up (left) and for the set-up with lead blanket (right)
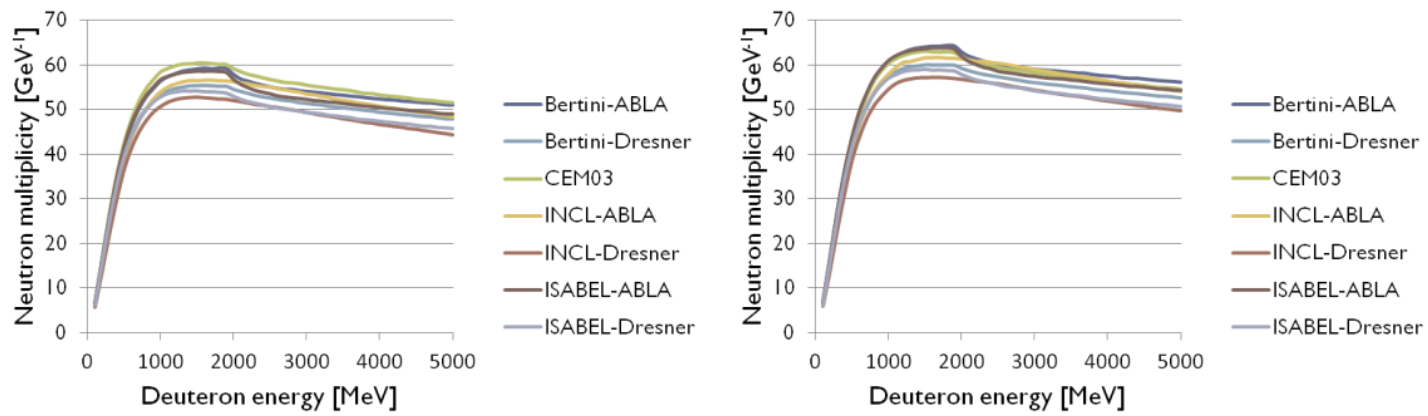

Fig. 4. Neutron multiplicity per $\mathrm{GeV}$ of primary beam energy for the bare set-up (left) and for the set-up with lead blanket (right) 


\section{Comparison of experimental values with calculation results}

\subsection{Measurement of neutron field}

The spatial distribution of the neutron field inside the set-up was measured by aluminium activation detectors. The activation samples were placed in the gaps between the sections on the detector plates in positions at distances of $0,-40,-80$ and $-120 \mathrm{~mm}$ from the centre on the target axis in the $-\mathrm{Y}$ radial direction. Al foils had square shape and size of $20 \times 20 \mathrm{~mm}$. The examined isotope ${ }^{24} \mathrm{Na}$ is produced both by deuterons from the primary beam and neutrons created inside the set-up by spallation and fission reactions. The amount of produced ${ }^{24} \mathrm{Na}$ radioisotope in ${ }^{27} \mathrm{Al}(\mathrm{d}, 3 \mathrm{p} 2 \mathrm{n}){ }^{24} \mathrm{Na}$ and ${ }^{27} \mathrm{Al}(\mathrm{n}, \alpha){ }^{24} \mathrm{Na}$ reactions was determined by means of gamma spectroscopic measurement. The obtained gamma spectra were analyzed and the net peak areas were calculated using the program DEIMOS32. All necessary spectroscopic corrections were applied during the evaluation of gained experimental data.

\subsection{Simulation of isotope production}

As input parameters of the beam characteristics for the simulation, the central part of the beam profile was fitted by Gaussian distribution and the horizontal and vertical FWHM and shift of the beam were taken from values measured in the experiment.

All principal particle types that can influence the reaction rates were taken into account in the simulations. Small volumes representing the specific detector positions during each irradiation were added to the Quinta model and neutron, proton, deuteron and charged pion spectral fluxes were calculated in these volumes using the MCNPX code. The major contribution to the yield is due to neutrons and protons, the influence of deuterons and charged pions is negligible except the case of the foils located at the beginning of the target which are directly hit by the primary deuteron beam.

To obtain the yields of isotopes, a convolution was made of the spectra for all considered particle types calculated in MCNPX (combination of models INCL4+ABLA with FLUKA for energies higher than $2.5 \mathrm{GeV}$ ) with reaction cross-sections for production of observed isotopes calculated in TALYS code (up to $200 \mathrm{MeV}$ in $1 \mathrm{MeV}$ energy bins) in combination with MCNPX (for energies higher than $200 \mathrm{MeV}$ up to the energy of the primary beam in $50 \mathrm{MeV}$ energy bins).

The yields of ${ }^{24} \mathrm{Na}$ produced in observed threshold reaction have common shapes in the 1 , 4 and $8 \mathrm{GeV}$ experiment respectively. The radial distribution of the yields shows almost an exponential decrease in transverse direction from the target axis to the edge. In longitudinal direction, the maximum production of ${ }^{24} \mathrm{Na}$ isotope for every experiment occurs near the target axis in the second gap between the blanket sections at $260 \mathrm{~mm}$ from the beginning of the target. The longitudinal distribution of the yields decreases sharply behind the second section towards the end of the blanket. 
The MCNPX code describes qualitatively well the shape of the longitudinal and radial distributions of the yields of threshold reactions. The difference between experimental and simulated yields of ${ }^{24} \mathrm{Na}$ is within $20 \%$ except for the $1 \mathrm{GeV}$ experiment, where the difference reaches $40 \%$ in certain cases. In the case of the 1 and $4 \mathrm{GeV}$ experiments from March 2012, the simulation underestimates the experimental values, while for the $4 \mathrm{GeV}$ experiment from December 2011 and $8 \mathrm{GeV}$ experiment from March 2012, the simulation overestimates the experimental values for most sample positions in the set-up.

The systematic uncertainty of the production rates mostly depends on the beam and detector displacement. Significant influence of the beam position on experimental results was observed in the activation detectors placed close to the target axis. The absolute values of the experimental yields depend on the beam intensity determination and detector displacement, whereas the results of the simulated yields depend on the beam input parameters (position, shape, parallelism with target axis) and on detector placement.
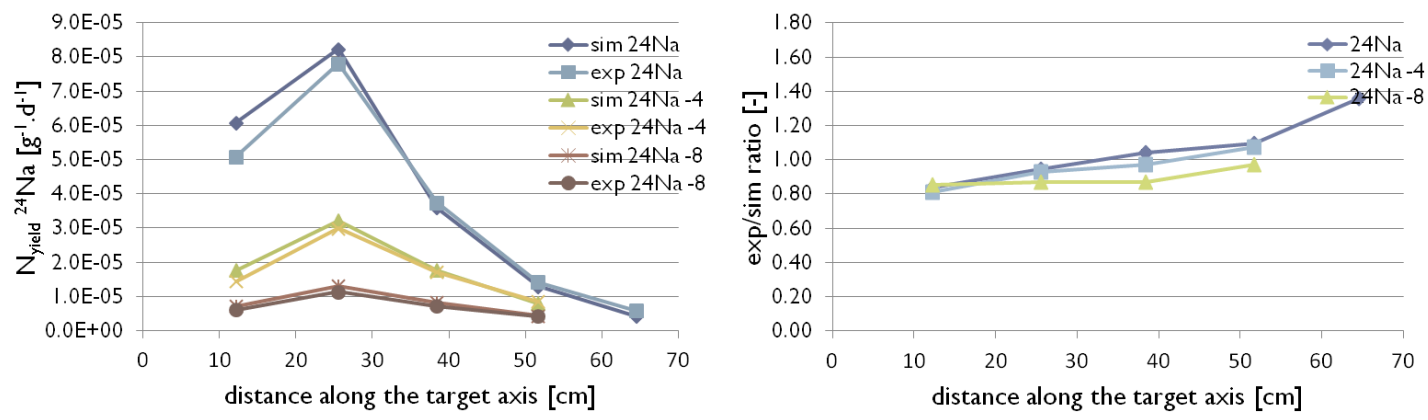

Fig. 5. Measured and calculated yields of observed ${ }^{24} \mathrm{Na}$ isotope in aluminium foils in longitudinal direction at $0,-40$ and $-80 \mathrm{~mm}$ below the target axis (left) and relative comparison of experimental and simulated yields (right), $4 \mathrm{GeV}$ experiment
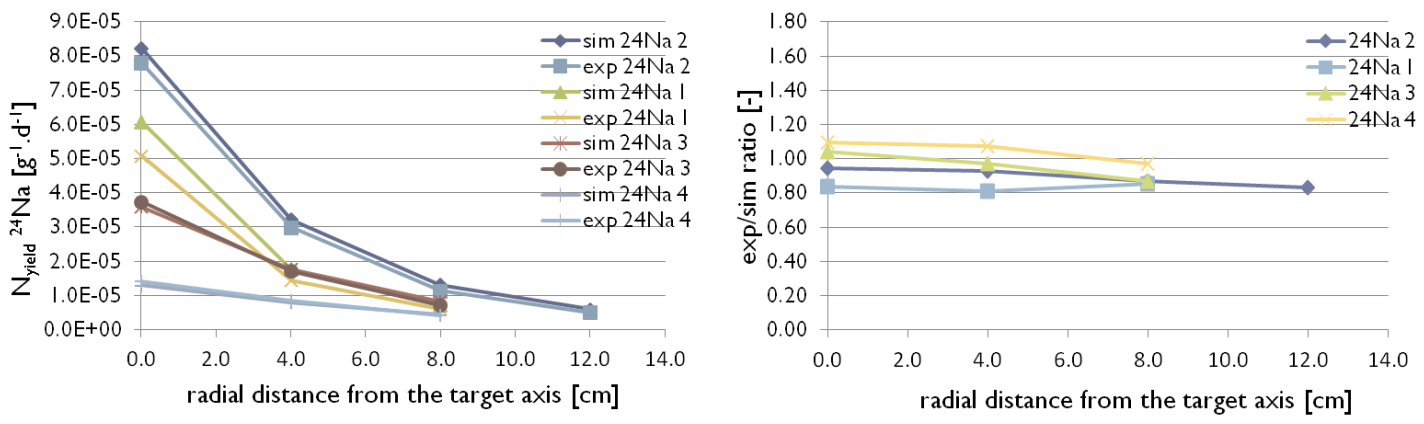

Fig. 6. Measured and calculated yields of observed ${ }^{24} \mathrm{Na}$ isotope in aluminium foils in radial direction downwards from the target axis in the 4 gaps between the set-up sections (left) and relative comparison of experimental and simulated yields (right), $4 \mathrm{GeV}$ experiment 

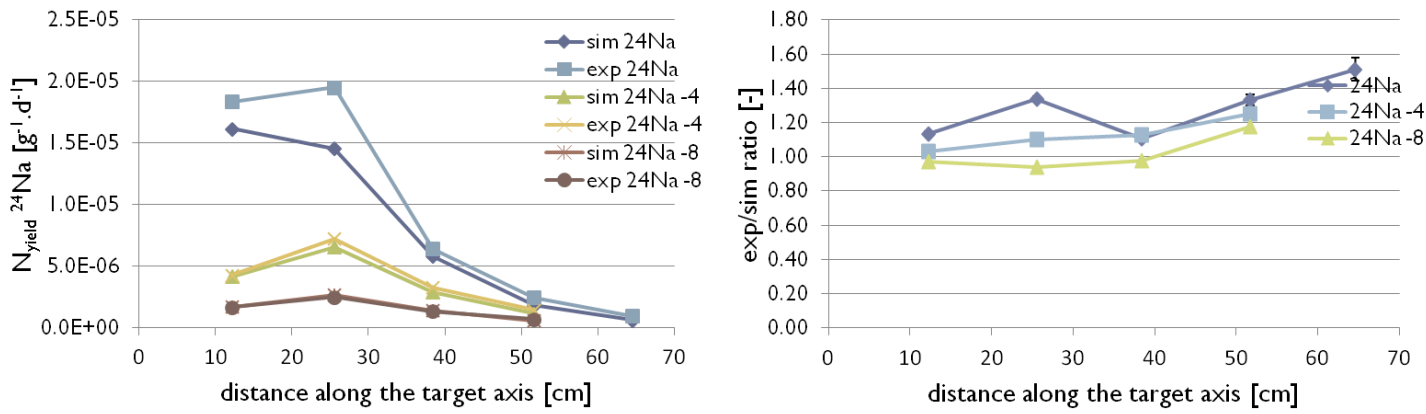

Fig. 7. Measured and calculated yields of observed ${ }^{24} \mathrm{Na}$ isotope in aluminium foils in longitudinal direction at $0,-40$ and $-80 \mathrm{~mm}$ below the target axis (left) and relative comparison of experimental and simulated yields (right), $1 \mathrm{GeV}$ experiment
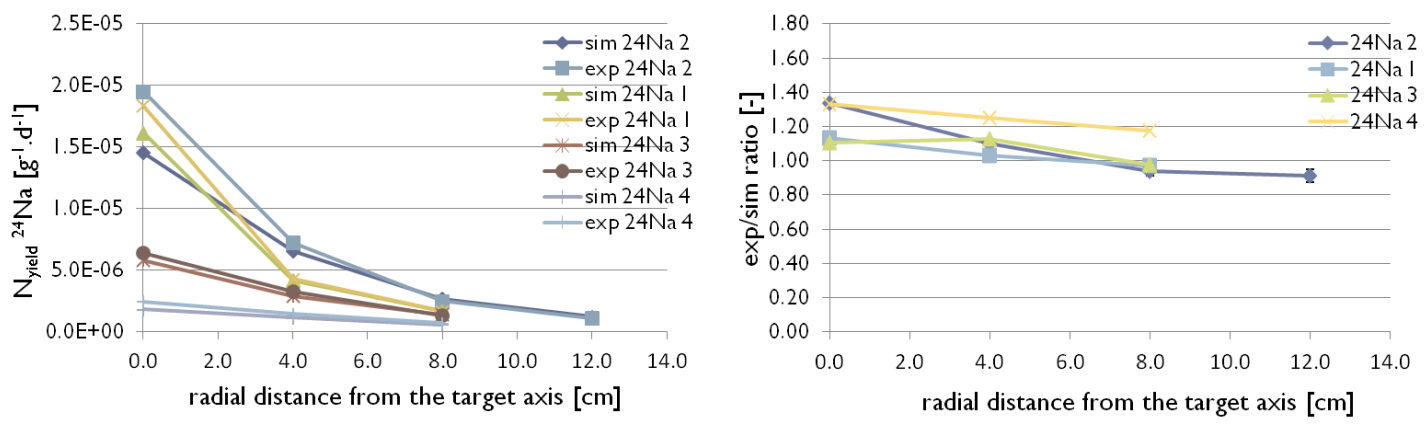

Fig. 8. Measured and calculated yields of observed ${ }^{24} \mathrm{Na}$ isotope in aluminium foils in radial direction downwards from the target axis in the 4 gaps between the set-up sections (left) and relative comparison of experimental and simulated yields (right), $1 \mathrm{GeV}$ experiment
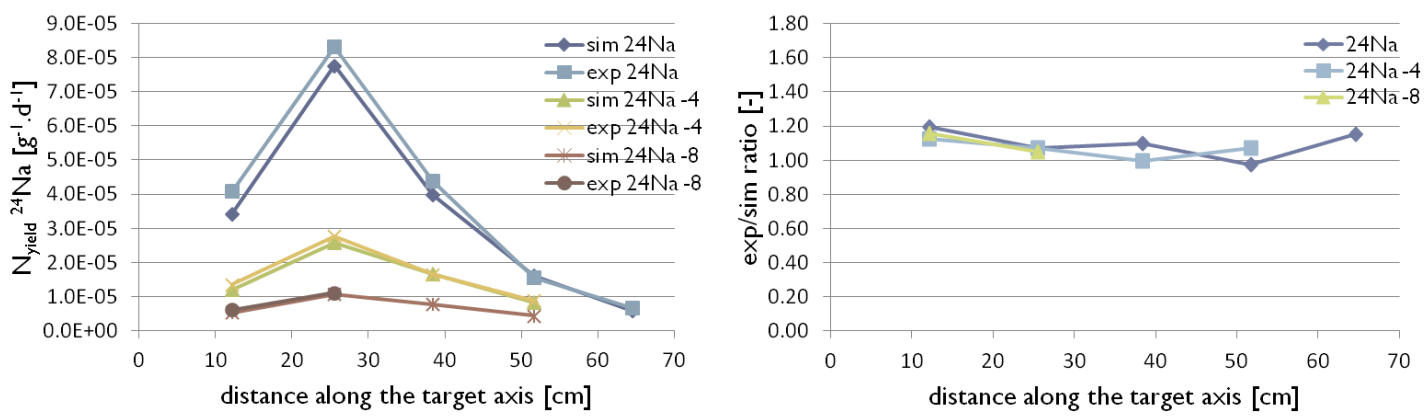

Fig. 9. Measured and calculated yields of observed ${ }^{24} \mathrm{Na}$ isotope in aluminium foils in longitudinal direction at $0,-40$ and $-80 \mathrm{~mm}$ below the target axis (left) and relative comparison of experimental and simulated yields (right), $4 \mathrm{GeV}$ experiment 

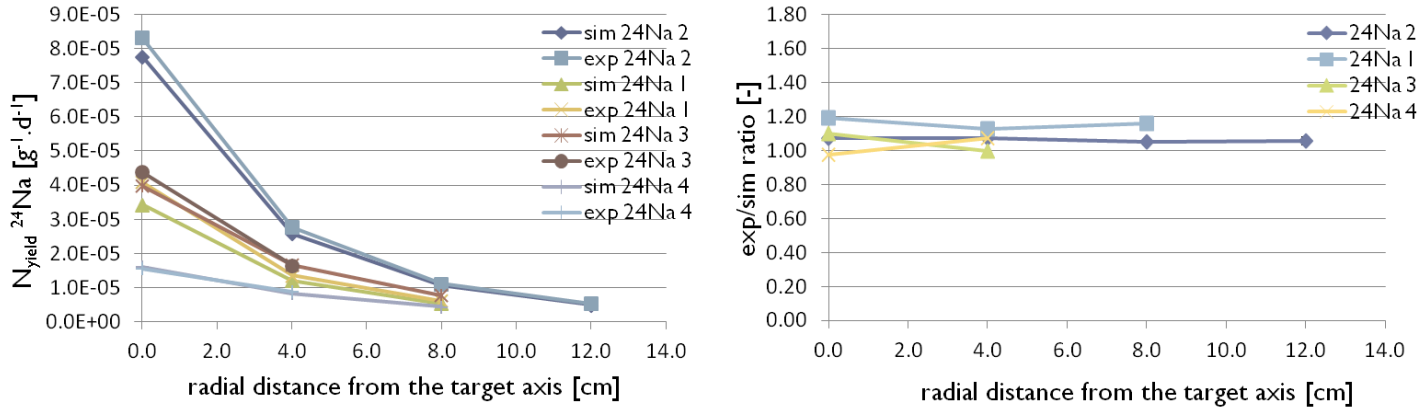

Fig. 10. Measured and calculated yields of observed ${ }^{24} \mathrm{Na}$ isotope in aluminium foils in radial direction downwards from the target axis in the 4 gaps between the set-up sections (left) and relative comparison of experimental and simulated yields (right), $4 \mathrm{GeV}$ experiment
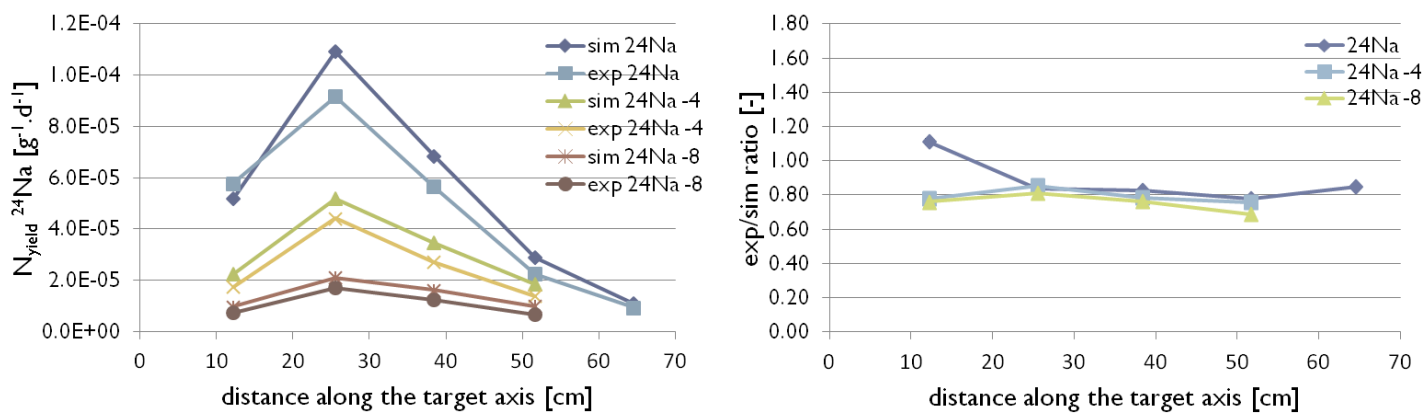

Fig. 11. Measured and calculated yields of observed ${ }^{24} \mathrm{Na}$ isotope in aluminium foils in longitudinal direction at $0,-40$ and $-80 \mathrm{~mm}$ below the target axis (left) and relative comparison of experimental and simulated yields (right), $8 \mathrm{GeV}$ experiment
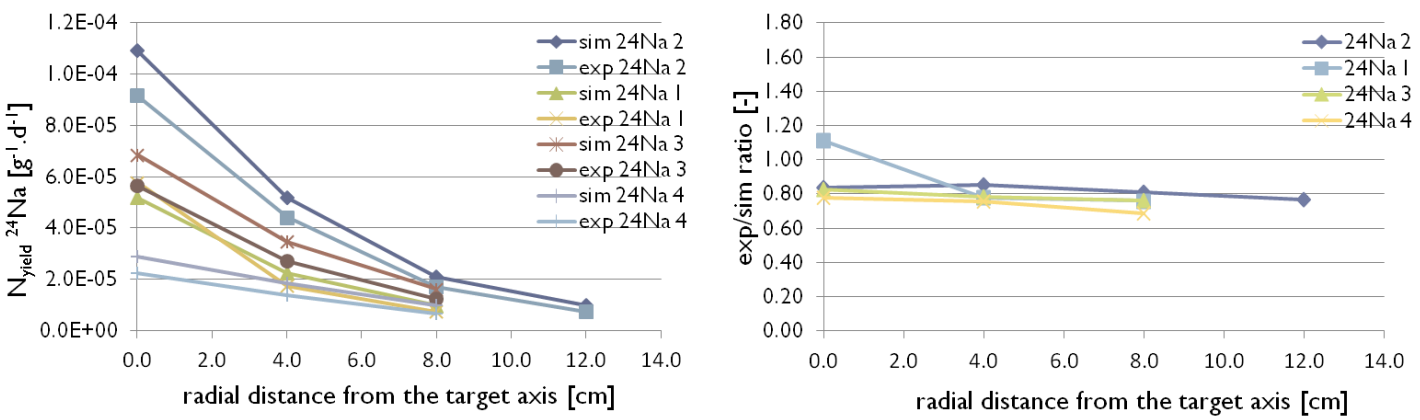

Fig. 12. Measured and calculated yields of observed ${ }^{24} \mathrm{Na}$ isotope in aluminium foils in radial direction downwards from the target axis in the 4 gaps between the set-up sections (left) and relative comparison of experimental and simulated yields (right), $8 \mathrm{GeV}$ experiment 


\section{Conclusion}

The Quinta set-up designed by the Energy plus Transmutation collaboration was used for the investigation of various fundamental aspects of Accelerator-Driven Systems concerning spallation and fission reactions, neutron production and transport, transmutation of radioactive materials, or measurement of delayed neutrons emerging from high-energy fission.

The MCNPX Monte Carlo code was applied to simulate the production and transport of neutrons and other secondary particles in the studied experimental set-up. In order to obtain calculated production rates of isotopes in different places of the set-up, the simulated spectra of neutrons, protons, deuterons and pions were folded with the cross-section for the specific reaction. The high-energy neutron flux in diverse places inside the system was measured by threshold reactions in aluminium activation detectors. The obtained experimental data were compared with the predictions of the MCNPX code. Relatively good agreement was found between experiment and simulation within roughly $20 \%$ difference in the majority of cases. There is no significant discrepancy between the experimental and simulated values of the yields. It can be concluded that the MCNPX simulation is in good qualitative agreement regarding the shape and intensity of produced neutron field with the measured data.

\section{References}

[1] Pelowitz D. B. et al., MCNPX Users's manual. Version 2.5.0, LANL report LA-CP-05-0369, 2005.

[2] A. J. Koning et al., TALYS 1.0, Proceedings of the International Conference on Nuclear Data for Science and Technology-ND 2007, 2007, 211-214.

[3] J. Frána, Program DEIMOS32 for Gamma-Ray Spectra Evaluation, J. Rad. Nucl. Chem., V. 257, No. 3 P. (2003) 583-587. 